\title{
"Fault Detection by Means of UL Passive Threat on Bearings
}

\author{
JOSÉ LUIS HERNÁNDEZ CORONA;ERNESTO MENDOZA VAZQUEZ, \\ JUAN EDUARDO ARROYO GAYOSSO,JUAN MARIO FLORES COLCHADO,JULIÁN TAPIA NIETO \\ ${ }^{1}$ Industrial Maintenance Engineering, Technological University of Tlaxcala
}

\begin{abstract}
In the mechanical systems it is important to monitor the state of the components, to avoid breakdowns and cause forced stops in the system. Some of the processes that exist in different companies do not have monitoring and / or vibrations that allow for better inspection quality within a process. The project carried out has the purpose of studying and measuring possible bearing or bearing failures with technological equipment such as EPOCH III from Parametric. Therefore, it helps in training the student in the use of these equipments in order to recognize a fault.
\end{abstract}

Keywords:- Sound, Ultrasound, Frequency, Wavelength, Bearing, Leak.

\section{INTRODUCTION}

Sound is the sensation that occurs when the vibration of waves that travel through the air or other medium, reaches the organ of hearing. Ultrasound corresponds to the spectrum greater than $20 \mathrm{kHz}$ that is not audible by the human ear. The airborne ultrasound technique uses the principle of ultrasonic waves propagated in the air and structures for the detection of faults. This technique is complementary to thermal and vibration analysis for the mechanical field, being in both cases the first instance of evidence of degradation, which in reliability is called the determination of the point ( $\mathrm{p}$ ) of probability of failure.

In the field of predictive maintenance, its popularity has been growing thanks to its ease of use, versatility and low implementation costs. At present, the use of this technique is incipient for Electric Power Systems (SEP), since it allows to carry out inspections without the electrical systems being directly intervened, being therefore safer than other techniques. (3)

This predictive maintenance technique is based on the detection of equipment failures, studying low-frequency sound waves produced by equipment that are not perceptible by the human ear. Sound whose frequency is above the pickup range of the human ear (20-20,000 Hertz) is considered ultrasound. Almost all mechanical frictions, electrical arcs, and pressure or vacuum leaks produce ultrasound in a range of approximately $40 \mathrm{Khz}$. This $40 \mathrm{khz}$ frequency. It has characteristics that are very useful in Predictive Maintenance, since the sound waves are short in length and rapidly attenuate without producing rebound. To apply it effectively and obtain representative and valid conclusions, it is necessary to know the machine well and select the most suitable analyzer. Ultrasound detection is a very useful technique in Predictive Maintenance because it is used to detect the exact place where some type of breakdown or failure is occurring. For this reason, in every industry this detection technique is currently used in Predictive Maintenance in production plant equipment, taking advantage of the properties of sound waves. [ 5]

And it is that the ultrasound locates exactly those areas where there are incipient problems. All this allows the adequate scheduling of the predictive maintenance of the equipment without hindering the normal development of the company.

It should be known that all mechanical and electrical problems, as well as vacuum or pressure leaks generate ultrasonic waves. These detect by means of meters in order to locate the problem and take corrective actions for its solution.

So, to detect ultrasound, an instrument called an ultrasound detector is used, designed to capture ultrasonic waves and convert them into signals with frequencies within the range of human hearing.

This device has the necessary technology so that once the waves are converted, they can be heard through headphones or viewed on a display. All by increasing its intensity

That is why the use of ultrasound in Predictive Maintenance is important, which is an important tool for the development of the industry. Because it allows detecting and locating equipment problems long before they cause interruptions, leading to large economic losses. [ 6]

In the different production processes, we always find the trend towards continuous improvement in production processes, storage, quality control, maintenance, among others, and new technologies are used to achieve these ends. Non-destructive testing is a very important part of quality control and represents a complement to other established methods in maintenance tasks in a certain plant. This technique can be applied in a sampling for an individual investigation or it can be used to inspect all the material in a quality control system. Given their versatility, these tests can be used in a wide variety of industrial applications. [3] 
This method studies low-frequency sound waves produced by equipment that are not perceptible to the human ear.

Ultrasound allows:

$>$ Detection of friction in rotating machines.

$>$ Detection of failures and / or leaks in valves.

$>$ Detection of fluid leaks.

$>$ Vacuum losses.

$>$ Detection of "electric arc".

$>$ Verification of the integrity of joints in watertight enclosures.

With these characteristics, it is used in Predictive Maintenance, since the sound waves are of short length, rapidly attenuating without producing rebounds. For this reason, ambient noise, no matter how intense, does not interfere with the detection of ultrasound. In addition, the high directionality of the ultrasound at $40 \mathrm{Khz}$. allows quickly and accurately the location of the fault. The application of ultrasound analysis is essential especially in the detection of existing faults in rotating equipment that rotates at 174 speeds lower than 300 RPM, where the vibration measurement technique becomes an inefficient procedure. So, ultrasound measurement is sometimes complementary to vibration measurement, which is used efficiently on rotating equipment rotating at speeds above 300 RPM.

\section{ULTRASOUND APPLICATION}

Ultrasound detection is a predictive maintenance technique that takes advantage of the properties of sound waves to detect problems in industrial plant equipment quickly, accurately and safely.

\section{Physical Principle:}

Sound is made up of a set of longitudinal mechanical waves produced by the vibration of objects and propagated through an elastic medium. These types of waves can stimulate the human ear and generate a sound sensation.

The characteristics of a sound wave are [1]:

- Cycle: It is the complete movement of the wave.

- Length: It is the distance necessary to complete a cycle. It is usually denoted as $\lambda$.

- Amplitude: It is the maximum value in positive and negative sense that the sound wave reaches.

- Frequency: It is the number of cycles per unit of time.

Ultrasound have one disadvantage of being dependent on the operator, which must have extensive knowledge of the physical principles of ultrasound, be familiar with the operation of equipment and ultrasonic measurement levels at full operation. The level of knowledge sought is high, for which desirable knowledge is required to be able to interpret the results in real time and recognize artifacts and thus avoid erroneous diagnoses and unnecessary procedures . [7]
Figure 1 shows a sound wave as a function of time with some characteristics. [two]

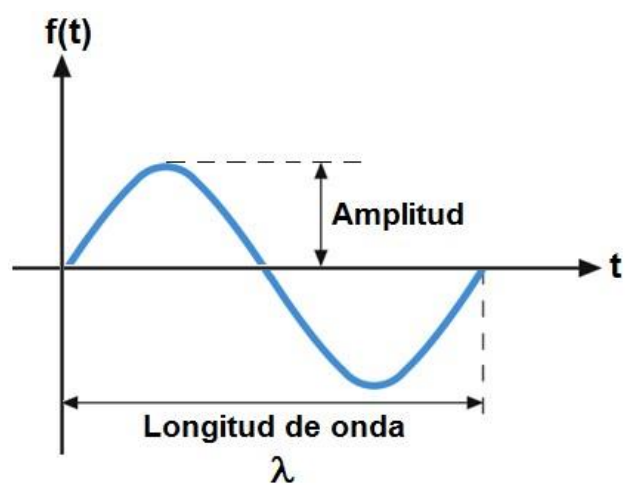

Fig 1:- Characteristics of a sound wave.

The knowledge of the ranges of the ultrasonic waves in mechanics will be used for the detection of industrial failures, for this work its use is exclusive to the detection of failures in bearings, not forgetting that some main applications are in the detection of leaks that represents an excellent option to reduce operating and maintenance costs. In many industrial facilities, Ultrasound detection instruments are used for safety, environmental protection, energy conservation programs and / or quality assurance .

Compressed air: Avoid energy losses by repairing your leaks. The ultrasound frequency scale is shown below, see figure 2 .

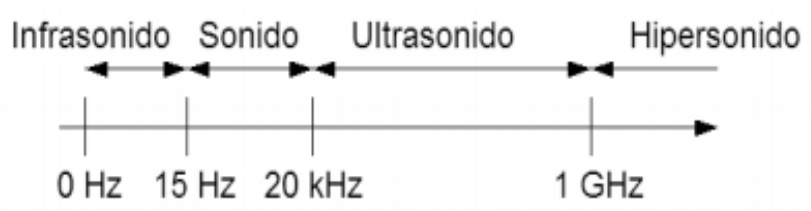

Fig 2:- Frequency scale

\section{Ultrasound Detection Technique:}

Ultrasound detection is a technique used in industrial maintenance based on the study of high frequency sound waves that are produced in equipment when something abnormal is happening.

This tool is based on the fact that friction forces, electrical discharges, and pressure or vacuum losses in plants generate high-frequency, short-length sound waves and rapid energy loss, which makes it possible to accurately locate problems. equipment before failures occur that interrupt the normal development of the production plant.

To detect ultrasound, an instrument called an ultrasound detector is used which is designed to capture ultrasonic waves and convert them into signals with frequencies within the range of human hearing. This device has the necessary technology so that once the ultrasound waves are converted, they can be heard through headphones or viewed on a display by increasing their intensity. 
Ultrasound detectors are easy to use equipment, thanks to the directional behavior of the sound, the operator can verify any area by locating the source of the problem, which manifests itself as a much louder sound than at other points. These detectors have a frequency selector that allows the user to filter out ambient noise and hear the ultrasonic wave clearly.

The internal design of an ultrasonic detector is shown in Figure 3. [2]

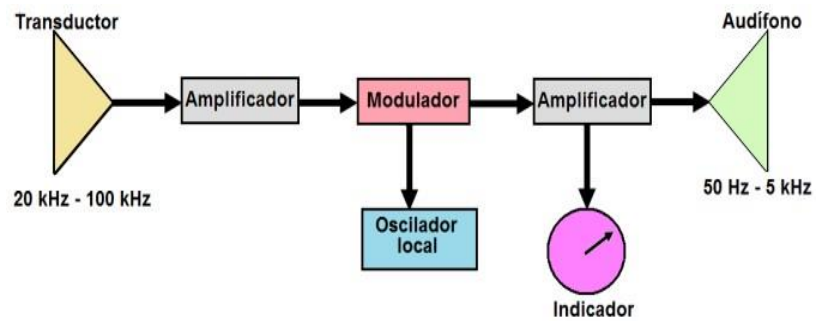

Fig 3:- Design of an Ultrasound Detector.

\section{METHODOLOGY}

The construction of the test module complies with the characteristics of a $1 \mathrm{Hp}$ motor, a speed variator, a pulley transmission system, a disc for simulation of imbalances, This will allow to study the frequencies of the bearings to the failures that are simulated at different speeds, In a second phase the detection of faults in bands as well as in the motor system is intended. See figure of proposed simulation bench.

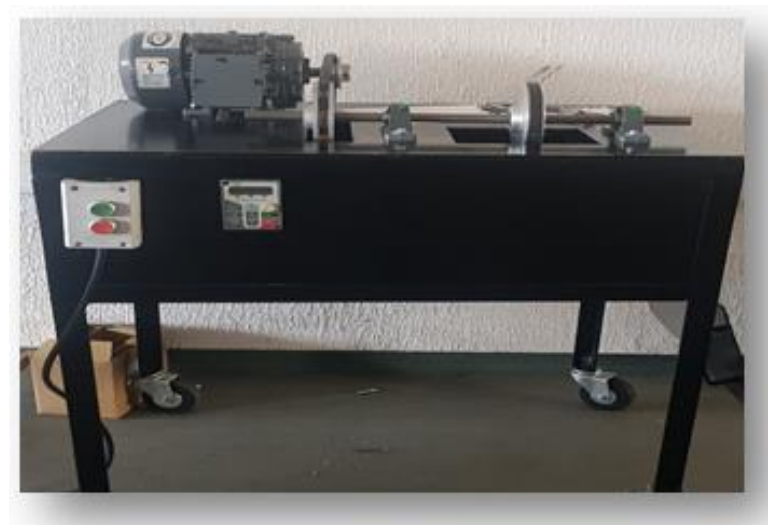

Fig 4:- Fault simulation module

El ultrasound is highly directional, which allows instruments to determine exactly where is happening the detected fault. These two capabilities, spectral diagnosis and directionality, make acoustic ultrasound an excellent option for the continuous monitoring of mechanical systems in continuous operation.

The right technology mind used in the right way and supported by international standards can contribute to the reliable operation of its facilities, improving availability and minimizing operating costs related to traditional maintenance practices.
Acoustic ultrasound also contributes to the safety of personnel by complementing the inspection methods by eliminating the risks of electric shock and flashing in the electrical part and in the mechanical part, bruises, loss of a member or destruction of equipment.

The development of learning models that contribute to a better application of techniques to identify failures from an early moment, before a more serious situation occurs, which allows planning to correct the failure.

The detection will be developed in each component where the bearings of the broken dynamic model are located, with an application such as the one proposed, the exact points where the failure occurs will be found.

It should be known that all mechanical and electrical problems, as well as vacuum or pressure leaks generate ultrasonic waves. These detect by means of meters in order to locate the problem and take corrective actions for its solution.

So, to detect ultrasound, an instrument called an ultrasound detector is used, designed to capture ultrasonic waves and convert them into signals with frequencies within the range of human hearing.

Ultrasound is a device that has the necessary technology so that once the waves have been converted, they can be heard through headphones or viewed on a display.

The level of understanding and management will depend on the level of specialization that is achieved.

\section{CONCLUSIONS}

To ensure the correct operation of the equipment in the industry, it is necessary to have the necessary and useful tools and techniques for its operation, as well as for its maintenance, the latter being the one that prolongs the useful life of the equipment.

The application of ultrasonic or sound waves allows better detection of mechanical failures in industrial environments, provides us with data for the early detection of problems, avoiding unexpected stoppages, as part of the techniques that integrate predictive maintenance.

\section{REFERENCES}

[1]. IDEAR. 2012. Vibracheck 200 mechanical vibration analyzer. Www.idearnet.com.ar .

[2]. Olarte C., Botero W. (2011) Ultrasound Detection: A Technique Used in Predictive Maintenance, Sciencia Et Technica, vol XVII, no. 47.

[3]. Pino C., (2015), Airborne Ultrasound in Power Electrical Systems, Electro Industria Technological Solutions Magazine for Mining, Energy and Industry, Editorial Microbyte Ltda 
[4]. PETERB, Erick. 2008. ULTRAPROBE 15000 , Instruction Manual 2008. http://dspace.espoch.edu.ec/handle/123456789/ $\underline{4571}$

[5]. Renovetec, (2014), Ultrasound Diagnostics, Renove Tecnología SL

[6]. Skalinet Corporation SAC, (2020), Ultrasound, a technique used in Predictive Maintenance, Journal of Maintenance Engineering, Asset Management and Productivity.

[7]. Vargas A., Amescua-Guerra L., Bernal Me. A., C. Pineda, (2008), Basic physical principles of ultrasound, s onoanatomía of the musculoskeletal system and sonographic artifacts, Medigraphic, Mexico 